\title{
Embedding Color Watermarks in Color Images
}

\author{
Chun-Hsien Chou \\ Department of Electrical Engineering, Tatung University, 40 Chungshan North Road, 3rd Section, Taipei 104, Taiwan \\ Email:chou@ttu.edu.tw
}

\author{
Tung-Lin Wu \\ Opto-Electronics \& System Laboratories, Industrial Technology Research Institute, Hsinchu, Taiwan \\ Email: tlwu@itri.org.tw
}

Received 17 May 2002 and in revised form 4 October 2002

\begin{abstract}
Robust watermarking with oblivious detection is essential to practical copyright protection of digital images. Effective exploitation of the characteristics of human visual perception to color stimuli helps to develop the watermarking scheme that fills the requirement. In this paper, an oblivious watermarking scheme that embeds color watermarks in color images is proposed. Through color gamut analysis and quantizer design, color watermarks are embedded by modifying quantization indices of color pixels without resulting in perceivable distortion. Only a small amount of information including the specification of color gamut, quantizer stepsize, and color tables is required to extract the watermark. Experimental results show that the proposed watermarking scheme is computationally simple and quite robust in face of various attacks such as cropping, low-pass filtering, white-noise addition, scaling, and JPEG compression with high compression ratios.
\end{abstract}

Keywords and phrases: robust and transparent watermarking, oblivious watermark detection, uniform color space, color quantization, just noticeable color difference.

\section{INTRODUCTION}

Digital watermarking is a technique that hides a piece of information in an original media for the purpose of copyright protection, integrity checking, or captioning $[1,2,3$, $4,5,6,7,8,9]$. The hidden information, or the so-called digital watermark, as usually represented by a sequence of random numbers or a recognizable binary pattern, should supply enough information for establishing rightful ownership or provide additional information about the original content. An effective watermarking scheme should meet certain requirements including transparency, robustness, security, unambiguity, and low-computational complexity. Depending on the application to be developed, the original data may or may not be used in the detection of watermarks. As considering the portability and availability of the original data, the oblivious (or blind) watermarking scheme without resorting to the original data is preferred. That is, a feasible image watermarking scheme should allow users to extract watermarks without referring to original images.

In the past few years, most researches focused on developing watermarking schemes for grayscale images. Only a comparatively small number of researches on color image watermarking can be found $[10,11,12,13,14,15,16,17]$. Some extend the algorithm used for grayscale images to the color case by marking the image luminance $[10,11]$. Fleet and Heeger [12] suggested to embed the watermark in the yellow-blue channel of the opponent-color representation of color images. Kutter et al. [13] embedded the watermark by modifying a selected set of pixels in the blue channel since the human eye is less sensitive to changes in this color channel. In [15], a repeated LSB-insertion watermarking technique for palette-based color images was proposed. In the method of quantization index modulation [16], each of the host signal is quantized by one of a number of quantizers, of which indices are used to carry the watermark information. A watermarking scheme based on ordered color quantization is proposed in [17]. It is found that the color quantization and processing in most of these proposed techniques are not optimized by taking properties of human visual perception into account. To gain high robustness and transparency in color image watermarking, the knowledge of human visual perception of color stimuli must be well utilized in designing watermark embedding/extraction algorithms.

In this paper, a spatial-domain color image watermarking scheme is proposed. Without resorting to the original 
image and with very little information as private key, the visually recognizable color watermarks can be detected by decoding each color pixel's quantization index which is modified to carry the watermark signal in the embedding process. The quantization index is modified in a way that results in a minimum color deviation. The color quantizer is so designed such that the quantization error and the distortion caused by the modification in quantization index will not be perceptible.

\section{COLOR QUANTIZATION}

Color is a visual perception of the light in the visible region of the electromagnetic wave spectrum incident on the retina. Since the retina has three types of photoreceptors that respond to different parts of the visible spectrum, three components are necessary and sufficient to specify a color. It has long been found that mean square error is a very poor measure of color difference in many tristimulus color spaces, such as RGB, CIEXYZ, YUV, and so forth. Color distributions in these tristimulus spaces are nonuniform in that the Euclidean distance between any two colors is usually not closely correlated with the associated perceptual difference. In case a nonuniform color space is uniformly quantized, the fixed color distance between any two colors of the quantized color space will result in large variation in perceptual difference, and perceptible distortion if the quantizer stepsize is large. In the proposed watermarking scheme, embedding and extraction of color watermarks are accomplished by color quantization. The image with the watermark embedded is actually the dequantization of a quantized image, of which quantization indices are disturbed by watermark information. To guarantee the transparency of the embedded watermark, the color difference between a pixel and its watermarked counterpart should be uniform and must not be perceptible throughout the whole image. To attain this goal, uniform quantization must be carried out in a uniform color space with the quantizer stepsize tuned to result in imperceptible color difference between any two adjacent colors in the quantized color space.

\section{PERCEPTUALLY LOSSLESS COLOR QUANTIZATION}

In this paper, color quantization is performed in the CIE-Lab color space where the color difference is more closely correlated with the perceptual difference. Any two colors with the same Euclidean distance in this space have approximately the same perceptual difference. A useful rule of thumb in this color space is that any two colors can be distinguished in a sense if their color distance

$$
\Delta E_{\text {Lap }}=\left[(\Delta L)^{2}+(\Delta a)^{2}+(\Delta b)^{2}\right]^{1 / 2}
$$

is greater than 3, the so-called just noticeable color difference (JNCD). The stepsize of the proposed uniform quantizer is thus determined in a way that the color difference between any two neighboring colors centroids should not be

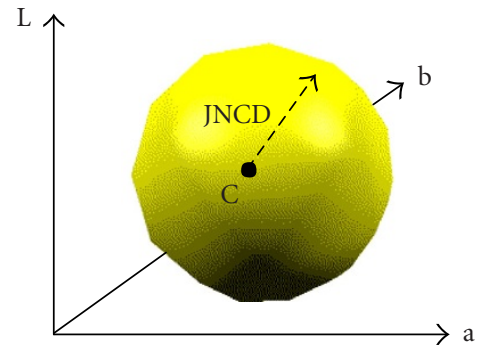

(a)

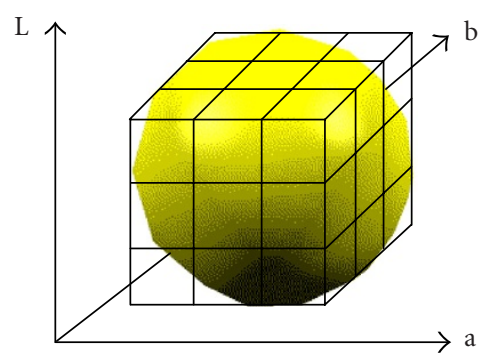

(b)

Figure 1: (a) The spherical subspace defined by color $C$ and the JNCD in the CIE-Lab space, within which all colors are perceptually indistinguishable from the color $C$; (b) the 27 uniform cubic subspaces that accommodate the spherical subspace.

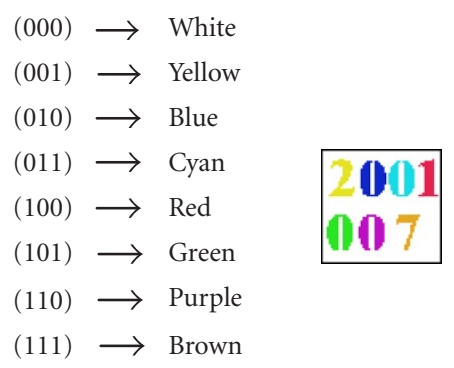

(a)

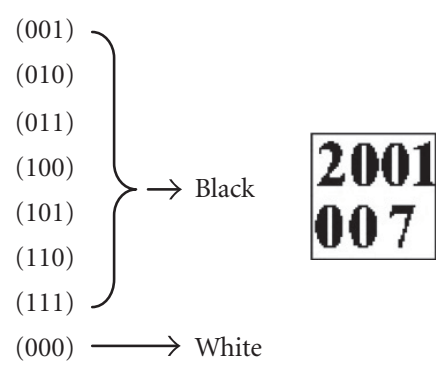

(b)

Figure 2: (a) The watermark represented by 8 different colors; (b) the watermark represented by two colors where the code (000) is assigned to represent color white and the rest of the codes color black. 


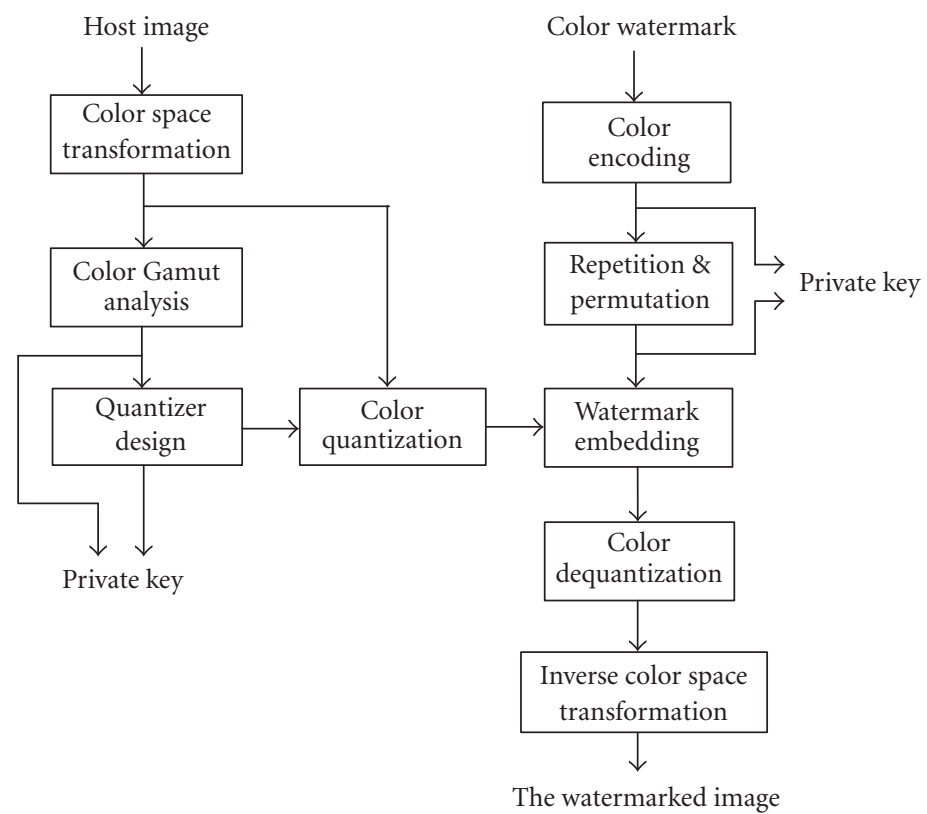

FIGURE 3: Watermark embedding process.

perceivable, or not to exceed the JNCD. By considering the masking effect mainly due to local variations in luminance magnitude, the quantizer stepsize can be set to be larger than JNCD.

Colors that are perceptually indistinguishable from a particular color in the uniform color space form a sphere with a radius equal to the JNCD (Figure 1a). As shown in Figure 1b, the spherical space can be approximated by a cubic space which can be further partitioned into $3 \times 3 \times 3$ uniform cubic subspaces. Colors within the centered cubic subspace are perceptually indistinguishable from colors within each of 26 peripheral cubic subspaces. Hence, as the uniform color space is partitioned into uniform cubic bins and the centroid of each bin is computed as a representative color, any color can be quantized and represented as a three-dimensional quantization index. A color pixel $P_{i}$ in the $R G B$ space can be transformed into a quantization index vector $\mathbf{q}_{\mathbf{i}}$ in the CIE-Lab space

$$
\begin{gathered}
\mathbf{q}_{\mathbf{i}}=\left(q_{L_{i}}, q_{a_{i}}, q_{b_{i}}\right)=\mathbf{Q}\left(L_{i}, a_{i}, b_{i}\right), \\
\left(L_{i}, a_{i}, b_{i}\right)=\mathbf{T}\left(R_{i}, G_{i}, B_{i}\right),
\end{gathered}
$$

where $\left(L_{i}, a_{i}, b_{i}\right)$ and $\left(R_{i}, G_{i}, B_{i}\right)$ are tristimulus values of the pixel $P_{i}$ in the CIE-Lab space and RGB space, respectively, while $\mathbf{T}$ denotes color transformation and $\mathbf{Q}$ uniform quantization. The transformation between the $R G B$ space and the CIE-Lab space is through the XYZ space. The RGB space is first converted to the $X Y Z$ space through a linear transformation

$$
\left[\begin{array}{l}
X \\
Y \\
Z
\end{array}\right]=\left[\begin{array}{lll}
0.490 & 0.310 & 0.200 \\
0.177 & 0.813 & 0.011 \\
0.000 & 0.010 & 0.990
\end{array}\right]\left[\begin{array}{l}
R \\
G \\
B
\end{array}\right] .
$$

Then, the XYZ space is converted to the CIE-Lab space through a nonlinear transformation

$$
\begin{aligned}
& L=116 f\left(Y / Y_{0}\right)-16, \\
& a=500\left[f\left(X / X_{0}\right)-f\left(Y / Y_{0}\right)\right], \\
& b=200\left[f\left(Y / Y_{0}\right)-f\left(Z / Z_{0}\right)\right],
\end{aligned}
$$

where

$$
f(x)= \begin{cases}\frac{1}{3 x}, & x>0.008856, \\ 7.787 x+\frac{16}{116}, & \text { otherwise }\end{cases}
$$

and $\left(X_{0}, Y_{0}, Z_{0}\right)$ represents the reference white. If the dimension of the cubic bin is appropriately set, the quantization error between a color and its counterpart after quantization and dequantization will not be perceivable, and so is the color difference between two adjacent colors in the quantized color space.

\section{SIGNAL SPACE FOR HIDING COLOR WATERMARKS}

By applying modulo-2 operation to each component of the three-dimensional quantization index, the quantization in$\operatorname{dex} \mathbf{q}_{\mathbf{i}}$ is mapped to a binary vector

$$
\hat{\mathbf{q}}_{\mathbf{i}}=\left(\hat{q}_{i L}, \hat{q}_{i a}, \hat{q}_{i b}\right)=\left(q_{i L} \bmod 2, q_{i a} \bmod 2, q_{i b} \bmod 2\right)
$$




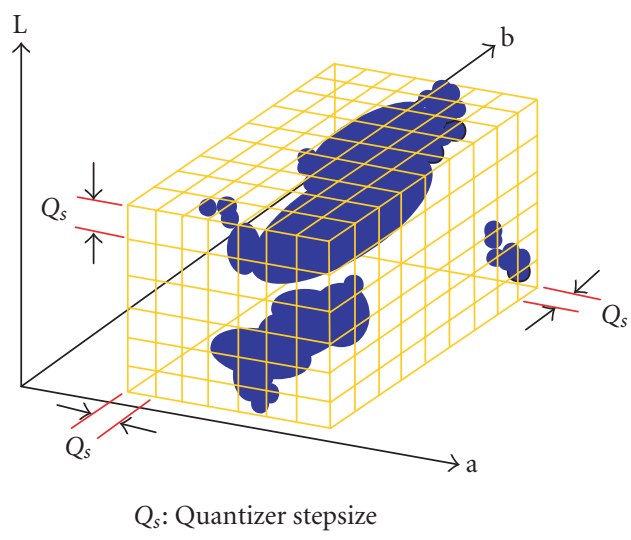

FIGURE 4: The quantizer design based on uniformly partitioning the subspace that best accommodates the color gamut of the host image.

in a three-dimensional binary space $\boldsymbol{\Phi}=\left\{\left(\phi_{1}, \phi_{2}, \phi_{3}\right): \phi_{k}=\right.$ 1 , or $0, k=1,2,3\}$. The space consists of eight binary vectors to which each centroid color of the uniform quantizer and its 26 adjacent centroid colors can be mapped. The mapping implies that any two vectors in $\boldsymbol{\Phi}$ are associated with two adjacent centroid colors of the uniform quantizer, which will be perceptually indistinguishable from each other if the quantization stepsize is small enough.

The eight binary vectors actually provide the space for carrying watermark information and can be used as codes for representing colors of the multilevel watermark. With multiple ways of color coding, the same watermark can have more than one representations at the same time. As shown in Figure 2, the watermark can be rendered by eight distinct colors, or as a binary image with the vector (000) being assigned to represent color white and the rest assigned to represent color black. With multiple codes being assigned to represent the same color, the watermark is expected to be more robust in that the color is enabled to tolerate a number of erroneous bits.

\section{WATERMARK EMBEDDING}

The process of embedding color watermarks in color images is described by the functional block diagram shown in Figure 3. The host image is first transformed into the CIELab color space where the color gamut of the host image is analyzed. With a quantizer stepsize, the rectangular subspace that best accommodates the color gamut is uniformly partitioned into cubic bins of identical dimension (Figure 4). The centroid of each cubic bin is then calculated as an output color of the quantization. One of eight corners of the rectangular subspace can be chosen as the reference origin which, together with the information of the subspace's dimension, is then taken as a part of the private key for specifying the color gamut. As the reference origin of the color gamut is determined, the centroid of each cubic bin can be addressed by a three-dimensional quantization index as described above. Before watermark embedding, one or more sets of color

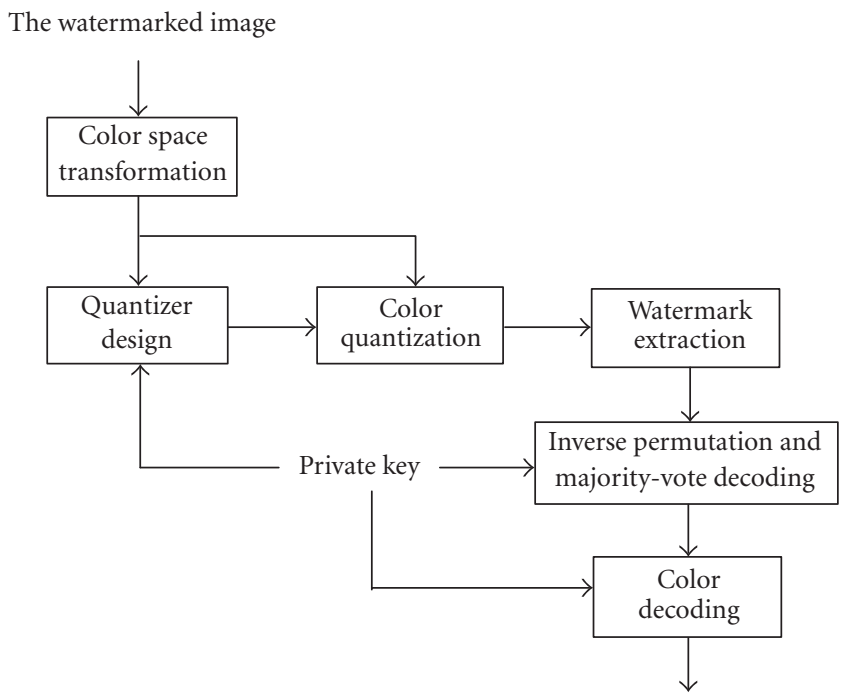

The extracted watermark

Figure 5: Watermark extraction process.

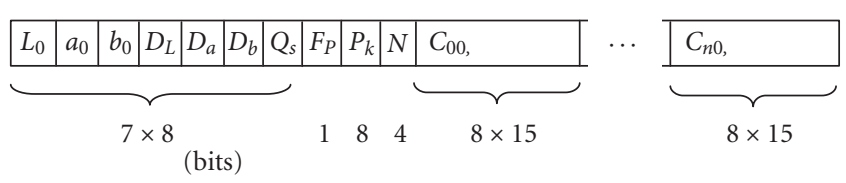

$L_{0}, a_{0}, b_{0}$ : reference origin of the color gamut

$D_{L}, D_{a}, D_{b}$ : dimension of the color gamut

$Q_{s}$ : quantizer stepsize

$F_{P}$ : flag for optional permutation

$P_{k}$ : permutation key

$N$ : number of color tables

$C_{i 0}, C_{i 1}, \ldots, C_{i 7}: 8$ colors in the $i$ th color table

FIGURE 6: Information contained in the private key.

tables are adopted as a part of the private key to render the watermark to multiple presentations, and the watermark is repeated to form a watermark image having the same dimension as the host image. In cooperating with the majority vote decision in the watermark extraction process, repeated embedding also functions to enhance the robustness of the watermark as subjected to various attacks that change tristimulus values of color pixels. Permutation is optionally applied to the watermark image for dispersing its spatial relationship, such that the watermark will not be easily removed by attacks that crop some parts of the image. A pseudorandom number traversing method is applied to both the row-number sequence $\{0,1, \ldots, H-1\}$ and the column-number sequence $\{0,1, \ldots, W-1\}$ with different seeds for random number generation to obtain a $2 \mathrm{D}$ sequence of coordinates, where $W$ and $H$ denote the width and height of the watermark image, respectively. The permutation is then accomplished by relocating the original pixels according to the new randomized 


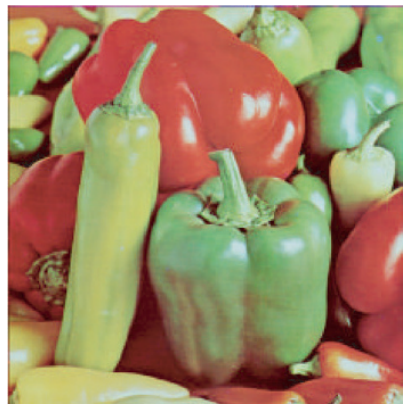

(a)

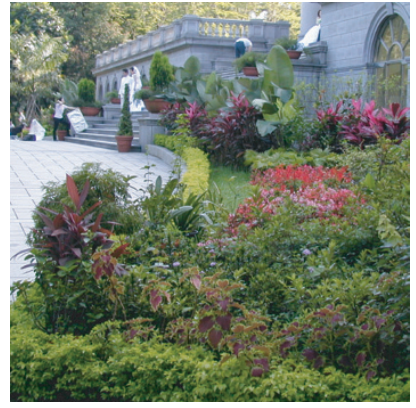

(b)

\section{1 007}

(c)

Figure 7: The color images (a) "PEPPER" and (b) “TTU," into which (c) the 8-color watermark to be inserted.

coordinates. The watermark signal $\mathbf{w}_{\mathbf{i}}=\left(w_{i 1}, w_{i 2}, w_{i 3}\right)$ is embedded into the color pixel $P_{i}$ by modifying its quantization index $\mathbf{q}_{\mathbf{i}}$ in the following way:

$$
\mathbf{q}_{\mathbf{i}}^{\mathbf{w}}= \begin{cases}\mathbf{q}_{\mathbf{i}}, & \text { if } d\left(\hat{\mathbf{q}}_{\mathbf{i}}, \mathbf{w}_{\mathbf{i}}\right)=0, \\ \mathbf{q}_{\mathbf{i}}+\left(v_{i 1} \cdot\left(\hat{q}_{i L} \oplus w_{i 1}\right), v_{i 2} \cdot\left(\hat{q}_{i a} \oplus w_{i 2}\right),\right. & \\ \left.v_{i 3} \cdot\left(\hat{q}_{i b} \oplus w_{i 3}\right)\right), & \text { if } d\left(\hat{\mathbf{q}}_{\mathbf{i}}, \mathbf{w}_{\mathbf{i}}\right) \neq 0,\end{cases}
$$

where $d\left(\hat{\mathbf{q}}_{\mathbf{i}}, \mathbf{w}_{\mathbf{i}}\right)$ is the Hamming distance between vectors $\hat{\mathbf{q}}_{\mathbf{i}}$ and $\mathbf{w}_{\mathbf{i}}$, and $\boldsymbol{\nu}_{\mathbf{i}}=\left(v_{i 1}, v_{i 2}, v_{i 3}\right)$ is a random vector with $v_{i k}=1$, or -1 for $k=1,2,3$. The modification, if required, is made such that quantization index is mapped to a binary vector identical to $\mathbf{w}_{\mathbf{i}}$. That is,

$$
\hat{\mathbf{q}}_{\mathbf{i}}^{\mathbf{w}}=\left(q_{i L}^{w} \bmod 2, q_{i a}^{w} \bmod 2, q_{i b}^{w} \bmod 2\right)=\mathbf{w}_{\mathbf{i}}
$$

The watermarked image, $\mathbf{I}^{\mathbf{w}}=\left\{P_{i}^{w}\right\}$, is then obtained by color dequantization and inverse color transformation

$$
P_{i}^{w}=\mathbf{T}^{-1}\left(Q^{-1}\left(\mathbf{q}_{\mathbf{i}}^{\mathbf{w}}\right)\right) .
$$

\section{WATERMARK EXTRACTION}

The process of watermark extraction is described by the functional block diagram shown in Figure 5, where the target image is first transformed to the CIE-Lab color space. The uniform quantizer used for watermark embedding is rebuilt by the private key information. As shown in Figure 6, the information organized in the private key contains the color gamut of the host image, quantizer stepsize, permutation key, and tables of colors for multiple representations. The number of bits required for representing these watermarking parameters can be as minimal as 189 bits. Each color pixel $P_{i}^{w}$ of the watermarked image is then quantized by the uniform quantizer

$$
\mathbf{q}_{\mathbf{i}}^{*}=\mathbf{Q}\left(\mathbf{T}\left(P_{i}^{w}\right)\right) .
$$

The watermark signal, $\mathbf{w}_{\mathbf{i}}^{*}$, carried by the quantization index is decoded by applying modulo-2 operation to each component of the quantization index

$$
\mathbf{w}_{\mathbf{i}}^{*}=\left(q_{i L}^{*} \bmod 2, q_{i a}^{*} \bmod 2, q_{i b}^{*} \bmod 2\right) .
$$

The image of the extracted watermark $\left\{\mathbf{w}_{\mathbf{i}}^{*}\right\}$ is then optionally arranged by the inverse permutation $\mathbf{R}$ and decoded by majority-vote decision $\mathbf{M}$ for obtaining the final watermark

$$
\tilde{\mathbf{W}}=\mathbf{M}\left(\mathbf{R}\left(\left\{\mathbf{w}_{\mathbf{i}}^{*}\right\}\right)\right) .
$$

Then, according to color tables found in the private key, the watermark is rendered to different representations. The intelligibility of the extracted watermark can be enhanced if some appropriate sets of colors are chosen for representing the multilevel watermark.

\section{EXPERIMENTAL RESULTS}

The performance of the proposed watermarking scheme in robustness and transparency is evaluated by attacking the watermarked image with various attacks listed in the Checkmark package [18] such as Gaussian noise addition, low-pass filtering, lossy JPEG compression, and geometric attacks including cropping, scaling, and rotation. Color images of size $512 \times 512$ are used as host images where each pixel is represented by 24 bits in the $R G B$ space, while the watermark image of size $128 \times 128$ contains visually recognizable patterns having at most 8 different colors. By taking the masking effect into account, the stepsize of the uniform quantizer designed in the CIE-Lab space is set to two times of the JNCD. In the simulation, attacks on the watermarked image are performed in the $R G B$ space. In Figure 7, an 8color watermark is embedded into two color images. From the watermarked images shown in Figure 8, the embedded watermark is visually transparent, and low values of PSNR indicate that the modification due to watermark embedding does not result in obvious and perceptible distortion. The extracted watermarks remain intact if no attack is performed on the watermarked images. Figure 9 shows the watermarks extracted from watermarked images which are attacked by 


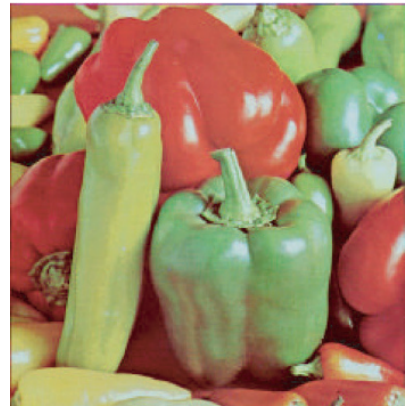

(a)

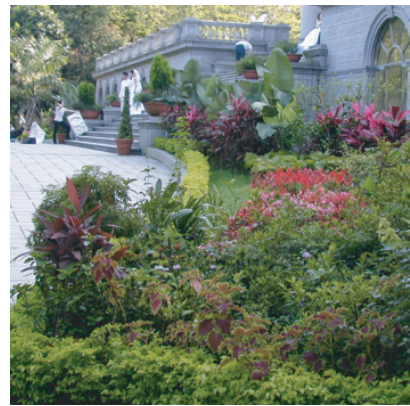

(b)

FIgURE 8: The watermarked images (a) "PEPPER" (PSNR = 33.35 dB) and (b) "TTU" (PSNR = 34.47), in which the color watermark of Figure $7 \mathrm{c}$ is embedded.

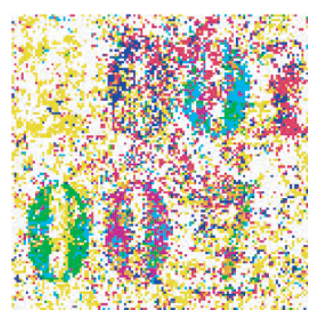

(a)

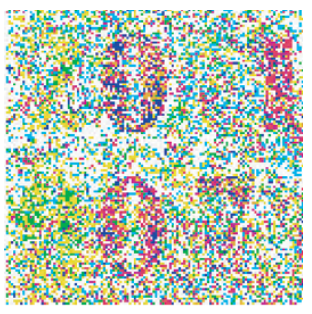

(b)

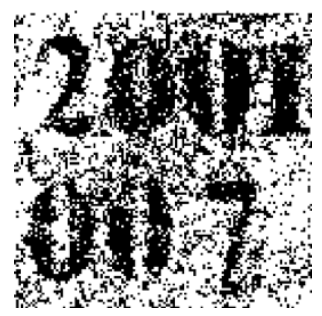

(c)

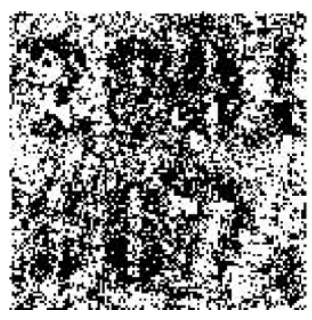

(d)

FIGURE 9: (a) The watermark extracted from the low-pass filtered watermarked image "PEPPER" (Normalized Correlation, NC = 0.539); (b) the watermark extracted from the low-pass filtered watermarked image "TTU" (NC = 0.423); (c) the binary rendition of the extracted watermark in (a); and (d) the binary rendition of the extracted watermark in (b).

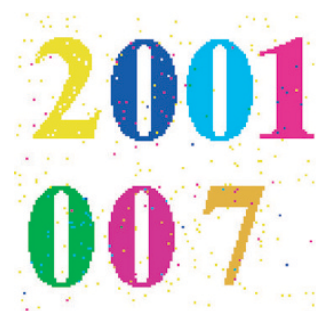

(a)

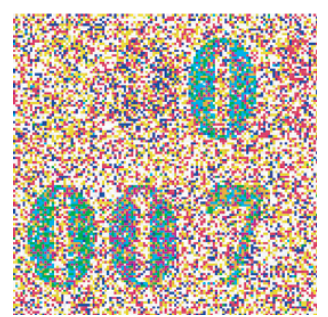

(b)

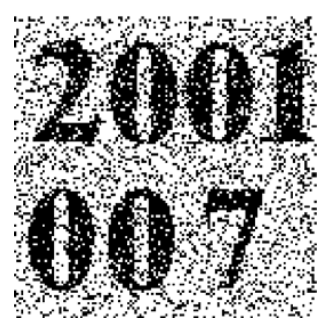

(c)

FIGURE 10: (a) The watermark extracted from the watermarked image "TTU" which is contaminated by zero-mean Gaussian white noises of variance $4(\mathrm{NC}=0.982)$; (b) the watermark extracted from the same watermarked image but contaminated by zero-mean Gaussian white noises of variance $25(\mathrm{NC}=0.36)$; and $(\mathrm{c})$ the binary rendition of the extracted watermark in $(\mathrm{b})$.

low-pass filterings. With the color coding schemes shown in Figure 2, the intelligibility of the watermark is improved by the binary rendition. Figure 10 shows the watermarks extracted from the watermarked images which are contaminated by zero-mean Gaussian white noises of variance 4 and 25. The watermarks extracted from watermarked images attacked by median filtering are shown in Figure 11. Since the local order statistics of the watermark image is largely destroyed by permutation, the permuted watermark is less robust to the median-filtering attack than the watermark with no permutation. Figure 12 shows that the embedded watermark is robust to the cropping attack even when the watermarked image is 50\% cropped, and that the permuted watermark is more robust than the watermark with no permutation. The watermarks extracted from the watermarked images which are scaled down and up by a factor of 4 are shown in Figure 13. From simulation results, it is found that the watermark can be perfectly recovered from the 


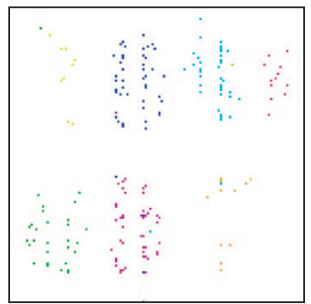

(a)

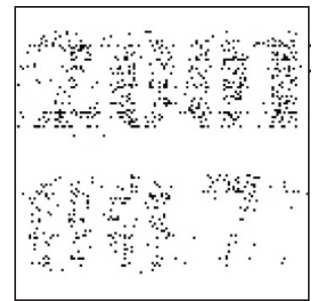

(b)

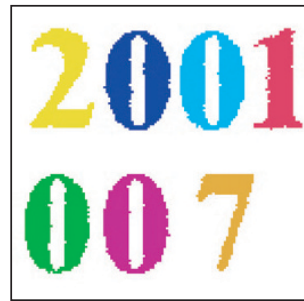

(c)

FIGURE 11: (a) The watermark extracted from the median-filtered watermarked image "TTU" with the watermark being permuted in the embedding process $(\mathrm{NC}=0.17)$; (b) the binary rendition of the extracted watermark in (a) $(\mathrm{NC}=0.253)$; and (c) the watermark extracted from the median-filtered watermarked image "TTU" with the watermark not being permuted in the embedding process $(\mathrm{NC}=0.993)$.

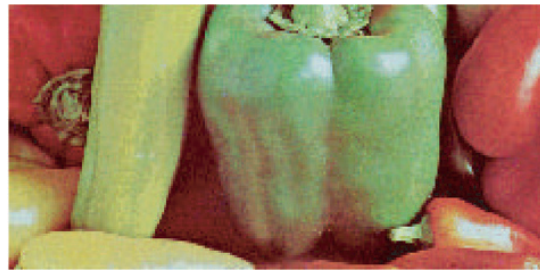

(a)

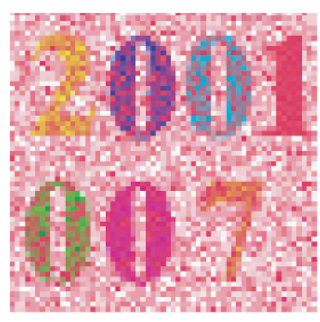

(b)

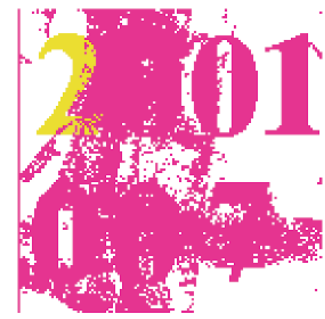

(c)
Figure 12: (a) The watermarked image which is $50 \%$ cropped; (b) the extracted watermark ( $\mathrm{NC}=0.553$ ) which is permuted in embedding process; and (c) the extracted watermark $(\mathrm{NC}=0.413)$ which is not permuted in embedding process.

watermarked images which are rotated by multiples of $90^{\circ}$, but it is not the case by other rotation angles. If the watermark image is not permuted in the embedding process, the watermark may survive the attack. The watermark image extracted from the watermarked image which is rotated by $30^{\circ}$ is shown in Figure 14. It demonstrates that the most part of the lwatermark image is destroyed by rotation, but the content of the watermark can still be identifiable by piecing up the parts that are readable. Figure 15 shows the watermarks extracted from watermarked images which are JPEGcompressed at different compression ratios. With multiplecolor tables, the extracted watermark can be rendered in multiple ways to give intelligible contents even if the com-

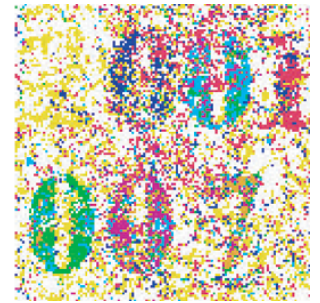

(a)

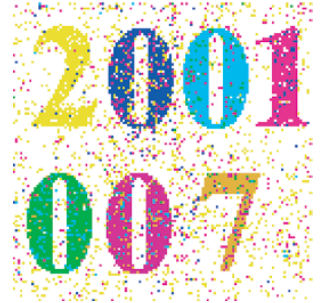

(b)
FIGURE 13: (a) The watermark extracted from the watermarked image "PEPPER" which is scaled down to a quarter of its original dimension $(\mathrm{NC}=0.536)$; (b) the watermark extracted from the watermarked image "PEPPER" which is scaled up to 4 times of its original dimension $(\mathrm{NC}=0.851)$.

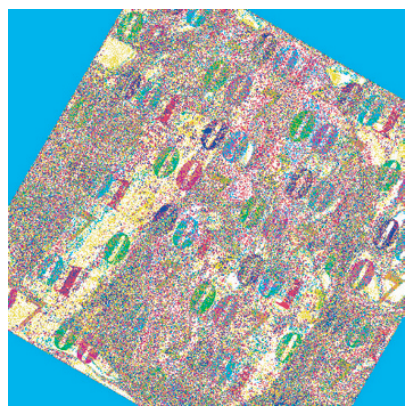

FIGURE 14: The watermark image extracted from the watermarked image "PEPPER" which is rotated by $30^{\circ}$.

pression ratio is as high as 27.5. As for the attack of color modification, the watermark may or may not be removed, depending on whether the amount of color pixels being modified dominates over the other, also both the original color and the modified color are mapped to the same binary vector as described in (6). From simulation results, the watermark can always survive the attack if half of all pixels is changed in colors in a random way. 


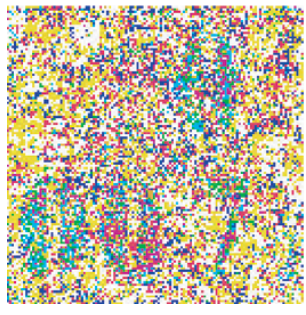

(a)

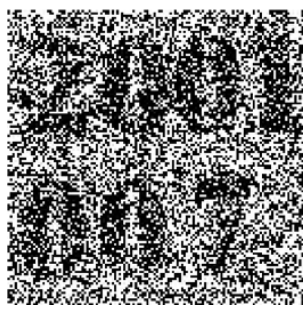

(b)

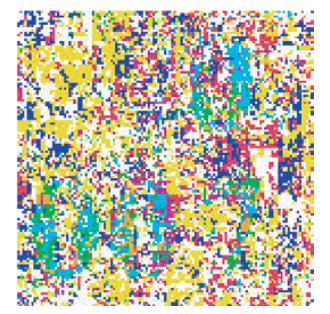

(c)

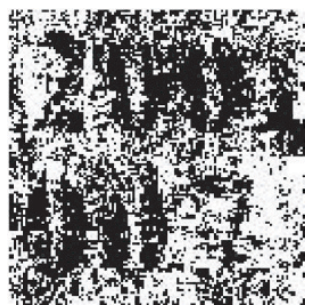

(d)

FIGURE 15: (a) The watermark extracted from the watermarked image "PEPPER" which is JPEG-compressed at a compression ratio of $12(\mathrm{NC}=0.439)$; (b) the binary rendition of the extracted watermark in (a) according to the color coding scheme of Figure 2a; (c) The watermark extracted from the watermarked image "PEPPER" which is JPEG-compressed at a compression ratio of 27.5 (NC $=0.343$ ); and (d) the binary rendition of the extracted watermark in (c) according to the color coding scheme of Figure 2 a.

\section{CONCLUSION}

In this paper, a color-image watermarking scheme that satisfies the requirements of transparency, robustness, and oblivious detection is proposed. Through color quantization, color watermarks are carried by the quantization indices of the host image in the uniform color space. Watermark transparency is achieved by perceptually lossless color quantization and modification in quantization indices. Watermark robustness is attained by repeated embedding, majority-vote decision, and multiple renditions of the watermark. Color watermarks are extracted without resorting to original images, but with a small amount of private-key information which can be as minimum as 189 bits. The proposed watermarking scheme also features its simplicity in computation and implementation. To further enhance the robustness of the color watermark in the color image, the same idea can be applied to images in the frequency domain. Embedding color watermarks in perceptually significant wavelet coefficients of the color image has already been under investigation.

\section{REFERENCES}

[1] R. G. van Schyndel, A. Z. Tirkel, and C. F. Osborne, "A digital watermark," in Proc. IEEE International Conference on Image Processing, vol. 2, pp. 86-90, Austin, Tex, USA, November 1994.

[2] I. Pitas, "A method for watermark casting on digital images," IEEE Trans. Circuits and Systems for Video Technology, vol. 8, no. 6, pp. 775-780, 1998.

[3] M. D. Swanson, M. Kobayashi, and A. H. Tewfik, "Multimedia data embedding and watermarking technologies," Proceedings of the IEEE, vol. 86, no. 6, pp. 1064-1087, 1998.

[4] M. Barni, F. Bartolini, V. Cappellini, and A. Piva, "Copyright protection of digital images by embedded unperceivable marks," Image and Vision Computing, vol. 16, no. 12-13, pp. 897-906, 1998.

[5] I. J. Cox, M. L. Miller, and J. A. Bloom, Digital Watermarking, Morgan Kaufmann Publishers, San Francisco, Calif, USA, 2001.

[6] F. Hartung and B. Girod, "Watermarking of uncompressed and compressed video," Signal Processing, vol. 66, no. 3, pp. 283-302, 1998.
[7] C. I. Podilchuk and W. Zeng, "Image-adaptive watermarking using visual models," IEEE Journal on Selected Areas in Communications, vol. 16, no. 4, pp. 525-539, 1998.

[8] C.-T. Hsu and J.-L. Wu, "Multiresolution watermarking for digital images," IEEE Trans. Circuits and Systems for Video Technology, vol. 45, no. 8, pp. 1097-1101, 1998.

[9] C.-S. Lu, S.-K. Huang, C.-J. Sze, and H.-Y. M. Liao, "Cocktail watermarking for digital image protection," IEEE Trans. Multimedia, vol. 2, no. 4, pp. 209-224, 2000.

[10] R. B. Wolfgang and E. J. Delp, "A watermarking technique for digital image: further studies," in Proc. IEEE International Conference on Imaging Science,System, and Technology, vol. 1, pp. 279-287, las Vegas, Nev, USA, 30 June-3 July 1997.

[11] I. J. Cox, J. Kilian, F. T. Leighton, and T. Shamoon, "Secure spread spectrum watermarking for multimedia," IEEE Trans. Image Processing, vol. 6, no. 12, pp. 1673-1687, 1997.

[12] D. J. Fleet and D. J. Heeger, "Embedding invisible information in color images," in Proc. IEEE International Conference on Image Processing, vol. 1, pp. 532-535, Santa Barbara, Calif, USA, October 1997.

[13] M. Kutter, F. Jordan, and F. Bossen, "Digital signature of color images using amplitude modulation," Journal of Electronic Imaging, vol. 7, no. 2, pp. 326-332, 1998.

[14] J. J. Chae, D. Mukherjee, and B. S. Manjunath, "Color image embedding using multidimensional lattice structure," in Proc. IEEE International Conference of Image Processing, pp. 460-464, Chicago, Ill, USA, October 1998.

[15] S. C. Pei and C. M. Cheng, "Pallete-based color image watermarking using neural network training and repeated LSB insertion," in Proc. the 13th IPPR Conference on Computer Vision, Graphics and Image Processing, vol. 1, pp. 1-8, August 2000.

[16] B. Chen and G. W. Wornell, "Quantization index modulation: A class of provably good methods for digital watermarking and information embedding," IEEE Transactions on Information Theory, vol. 47, no. 4, pp. 1423-1443, 2001.

[17] C. C. Tseng, C. J. Juan, and S. L. Lee, "Color image watermarking based on ordered color quantization," in Proc. the 13th IPPR Conference on Computer Vision, Graphics and Image Processing, vol. 1, pp. 458-466, August 2000.

[18] S. Pereira, S. Voloshynovskiy, M. Madueño, S. MarchandMaillet, and T. Pun, "Second generation benchmarking and application oriented evaluation," in Information Hiding Workshop III, pp. 340-353, Pittsburgh, Pa, USA, April 2001. 
Chun-Hsien Chou graduated from $\mathrm{Na}-$ tional Taipei Institute of Technology, Taipei Taiwan in 1979, and received the M.S. and Ph.D. degrees in electrical engineering from National Tsing Hua University, Hsinchu, Taiwan, in 1986 and 1990, respectively. In 1990, he joined the Department of Electrical Engineering at Tatung Institute of Technology, Taipei, Taiwan, as an Associate Professor. During the academic year from 1991

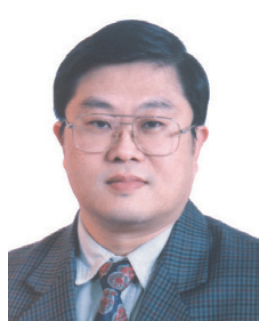
to 1992, he was a Postdoctoral Research member at AT\&T Bell Laboratories, Murray Hill, NJ. In 1996, he became a Professor at the Department of Electrical Engineering, Tatung University, Taipei, Taiwan. His current research areas include color models of the human visual system, perceptual coding of color images, streaming video coding, virtual reality, and digital watermarking techniques.

Tung-Lin Wu received the B.S. degree in electrical engineering from Tamkang University, Tamshui, Taiwan in 1999, and the M.S. degree in communication engineering from Tatung University, Taipei, Taiwan in 2001. He is currently working in Opto-Electronics \& Systems Laboratories of Industrial Technology Research Institute, Hsinchcu, Taiwan, as an Associate Engineer. His current research areas include color sig-

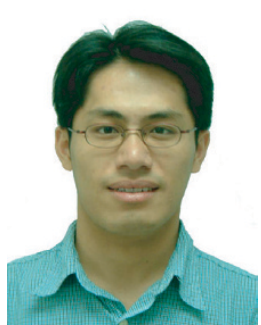
nal processing and image coding. 